\title{
Status and Perspectives of the COBRA-Experiment
}

\author{
Daniel MUENSTERMANN* on behalf of the COBRA collaboration ${ }^{\dagger}$ \\ CERN, CH-1211 Geneve 23, Switzerland \\ E-mail: Daniel.Muenstermannecern.ch
}

\begin{abstract}
The determination of two important properties of the neutrino is still pending: its rest mass and its nature - Dirac or Majorana particle. The observation of neutrinoless double beta decay would clarify both, but the expected half-life of more than $10^{25}$ years for this decay is an ambitious challenge.

Recently, first experiments have been commissioned that will be able to probe this magnitude of half-lifes. If they succeed, it will be important to verify the results using different isotopes. If they do not succeed, new experimental approaches are required.

The COBRA experiment is an excellent candidate for both: it uses CdZnTe room-temperature semiconductor detectors that contain several double beta decay candidate isotopes, among them also $\beta^{+}$emitters and two of the most promising isotopes, Te-130 and Cd-116. Cd-116 has a decay energy well above the most energetic naturally occurring gamma lines reducing its background drastically compared to isotopes with lower Q-values. In addition, CdZnTe can be used as a solid-state TPC combining the large advantages of a source-equals-detector approach with the possibility of decay identification via particle track reconstruction.

The COBRA collaboration comprises 12 institutes from 8 countries and currently operates detectors with two readout techniques: The commercially available Co-Planar Grid (CPG) technology was developed specifically for CdZnTe and allows the operation of fairly large crystal sizes of the order of some $\mathrm{cm}^{3}$ with only one readout channel. It reaches energy resolutions better than $2 \%$ FWHM at $662 \mathrm{keV}$. COBRA operated several R\&D-arrays of CPG-type detectors in ultra-low background mode at the Italian underground laboratory LNGS. Half-life limits above $10^{20}$ years for several isotopes and decay modes have been determined with these setups. New readout electronics optimized for low-rate spectroscopy and allowing background reduction by pulse-shape analysis was developed.

Using semiconductor pixel detectors is unique for a double-beta decay experiment. Three different pixelated $\mathrm{CdZnTe/CdTe}$ detector types have been operated in an ultra-low background environment and have demonstrated the power of background suppression with this detector type by enabling fiducial cuts, vertexing and coincidence techniques.
\end{abstract}

The 2011 Europhysics Conference on High Energy Physics-HEP 2011,

July 21-27, 2011

Grenoble, Rhône-Alpes France

\footnotetext{
*Speaker.

${ }^{\dagger}$ http://www.cobra-experiment.org
} 


\section{Double Beta Decay}

Some (even-even) nuclei cannot undergo beta decay for energetic reasons. However, they can decay to the next even-even nucleus by two consecutive decays, a second-order process and hence heavily suppressed: Half-lives are of the order of $10^{20}$ years. In addition to this standard model compliant process which is associated with the emission of two neutrinos $(2 v \beta \beta)$, one can also envisage a combination of beta decay and inverse beta decay, if the neutrino is its own anti-particle: Neutrinoless Double Beta Decay $(0 v \beta \beta)$.

Double beta decay can occur in the flavours $\beta^{-} \beta^{-}, \beta^{+} \beta^{+}, \beta^{+} / \mathrm{EC}$ and $\mathrm{EC} / \mathrm{EC}$ which have different experimental signatures. All neutrino-emitting decays (but EC/EC) will exhibit a continuous spectrum, but in case of neutrinoless decays, the electrons/positrons will carry the complete decay energy, hence the sum energy spectrum will be a line at the Q-value of the decay.

A line signature is very well visible experimentally, however, the expected half-lives of neutrinoless double beta decays are of the order of $10^{26}$ years so good background suppression is mandatory. While the half-lives scale with the effective Majorana neutrino mass, a measurement of angular distributions and/or of certain positron-emitting decays might help to disentangle the underlying mechanisms, e.g. right-handed weak currents.

\section{COBRA - Idea and Advantages}

Low-background spectroscopy based on semiconductor detectors takes advantage of the good intrinsic radiopurity of the base material yielding a rather low background. In addition, particle tracking and vertexing might be feasible in form of a solid-state TPC using pixel detectors which would allow for much improved background reduction and the measurement of angular distributions.

The idea of COBRA is to use an array of CdZnTe semiconductor detectors to search for neutrinoless double beta decays[1]. CdZnTe contains 9 isotopes with the potential to decay via DBD, among them Cd-116 with a Q-value above the highest naturally occuring background line, Te-130 with a natural abundance of $34 \%$ and Cd-106 which can decay via all $\beta^{+}$-modes.

Due to crystal growth limitations, the sizes of spectroscopy grade CdZnTe is currently limited to few $\mathrm{cm}^{3}$. To reach source masses of several $100 \mathrm{~kg}$, the operation of a large, 3-dimensional array with $\mathrm{O}\left(10^{4}\right)$ detectors is necessary. In CdZnTe, the mobility-lifetime product for holes is quite poor; therefore, readout schemes relying solely on electrons are utilised. COBRA investigates two of these, CPG-type and pixel readout.

\section{Co-Planar Grid Detectors}

One method to reach a single charge carrier sensitive device is the application of a Frisch-Gridlike approach proposed by Luke[2]. This readout scheme allows for quite large/thick detectors with sufficiently homogenious response and already the operation of first prototype arrays based on this technique yielded half-life limits above $10^{20}$ years[3, 4].

From the pulse shapes, also the interaction depth can be deduced allowing for fiducial cuts. While earlier results were relying on analogue signal subtraction, new readout electronics based 
on Flash-ADCs have been developed and installed allowing to study the pulse shapes. First results show that the energy resolution could be improved significantly up to $2 \%$ at $662 \mathrm{keV}$ and that the background could be further identified and reduced. Evaluation of the instrumentation of the boundary electrode to achieve discrimination power also with respect to the side surfaces is currently underway.

\section{Pixel Detectors}

Pixel detectors offer superior options for background reduction: Fiducial cuts to the side surfaces are possible simply based on excluding the outermost pixels. By instrumenting the cathode, also depth-sensing is feasible enabling 3-dimensional position sensing. This was first demonstrated in the COBRA low-background setup at the LNGS underground laboratory with a Polaris system (Z. He, U. of Michigan) which comprises a very large CdZnTe crystal ( 2 by 2 by $1.5 \mathrm{~cm}$ ) with 11 by 11 pixels. The pixels are small enough to enable electron-only readout, but large enough to collect all deposited charge in one (or two) pixels leading to superb energy resolution. Although the system was not specifically prepared for low-background operation, the ROI of Cd-116 could be cleared of any event by applying a ficucial cut which leads to a background level of less than 4 counts $/ \mathrm{keV} / \mathrm{kg} / \mathrm{y}$. A preliminary analysis leads to a half-life limit for Cd-116 of better than $10^{20}$ years with only $4.3 \mathrm{~kg} \mathrm{~d}$ of data, demonstrating the advantages of background suppression[5].

The second approach followed is that of a solid-state TPC: If the pixels are small enough (few hundred $\mu \mathrm{m}$ ), the tracks of the decay electrons are visible and clearly distinguishable from alpha and gamma background. By means of neural networks, a significant S/B improvement is also achievable for single-electron background. In spite of the $\Delta \mathrm{E} / \mathrm{E}$ deterioration due to manypixel events, the energy resolution of pair-production events at $1.59 \mathrm{MeV}$ was measured to be still better than $0.7 \%$ with a TimePix-based system. Methods to arrange many pixel detectors in a low-background compatible way are currently under investigation.

\section{Outlook}

To achieve sensitivities for neutrino masses of the order of $50 \mathrm{meV}$, it is necessary to probe half-lives of about $2 \cdot 10^{26}$ years which requires source masses of $\sim 400 \mathrm{~kg}$ CdZnTe enriched in the isotope of interest to $90 \%$. Such a setup would comprise e.g. 40 by 40 by 40 (i.e. 64000) CPG-type detectors of $1 \mathrm{~cm}^{3}$ size each or of an appropriate number of pixel detectors which would probably have larger surface $\left(2 \mathrm{~cm}^{2}\right)$, but less thickness ( 3 to $5 \mathrm{~mm}$ ). Detailed studies for scalable setups are ongoing.

\section{References}

[1] K. Zuber, Phys. Lett. B 519, 1 (2001)

[2] P. Luke, Appl. Phys. Lett. 65, 2884 (1994).

[3] H. Kiel, D. Muenstermann and K. Zuber, Nucl. Phys. A 723, 499 (2003)

[4] J. Dawson et al., Phys. Rev. C 80, 025502 (2009)

[5] O. Schulz, PhD thesis, TU Dortmund, 2011, https://eldorado.tu-dortmund.de/handle/2003/29108 